\title{
RDUS
}

Revue de DROIT

UNIVERSITÉ DE SHERBROOKE

Titre : $\quad$ LES MINORITÉS EN DROIT DES BIENS ET DES OBLIGATIONS AU QUÉBEC : QUELQUES ASPECTS

Auteur(s): $\quad$ Adrian POPOVICl

Revue : $\quad$ RDUS, 2003-2004, volume 34, numéro 1-2

Pages: $\quad 261-274$

ISSN : $\quad 0317-9656$

Éditeur : $\quad$ Université de Sherbrooke. Faculté de droit.

URI : $\quad$ http://hdl.handle.net/11143/12285

DOI : https://doi.org/10.17118/11143/12285 
Page vide laissée intentionnellement. 


\section{LES MINORITÉS EN DROIT DES BIENS ET DES OBLIGATIONS AU QUÉBEC : QUELQUES ASPECTS*}

par Adrian POPOVICI**

SOMMAIRE

I. Les minorités au sein de la société québécoise : l'apothéose

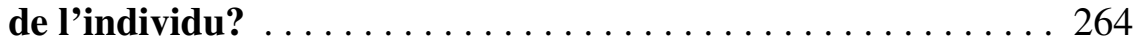

II. Les minorités au sein de groupements : la dictature de la majorité?

\footnotetext{
*. Exposé présenté aux Journées mexicaines de l'Association Henri Capitant à Mexico et Oaxaca du 18 au 25 mai 2002.

**. Professeur à la Faculté de droit de l’Université de Montréal.
} 

Le grand défi du présent rapport est d'abord la délimitation du sujet devant être traité. Il s'agit d'un aspect du droit relatif à des minorités en droit civil québécois, la partie qui traite des personnes et de la famille étant confiée à notre collègue Benoit Moore. De plus, la question des minorités est tout naturellement plutôt du domaine du droit public et du droit international... Il convient de ne pas empiéter sur ces terres et même d'éviter aussi de traiter des minorités en droit des affaires (sociétés commerciales), tout en respectant les choix et les balises établis par notre rapporteur général, le doyen Jacques Mestre. De surcroît, autant que faire se peut, il faut aussi éviter de faire double emploi avec les sujets des journées Henri Capitant de l'an dernier qui portaient sur la discrimination.

La notion de minorité présuppose deux groupes de personnes, le premier majoritaire, le second minoritaire. Il convient de se pencher sur la façon dont s'articule cette lapalissade dans le cadre du droit civil. Le droit privé se préoccupe principalement des droits et devoirs de l'individu, lequel, il est vrai, peut faire partie d'une minorité significative à l'intérieur de la société québécoise. Si le droit reconnaît et sanctionne ses droits individuels (dont son droit à l'égalité), n'est-ce pas clairement souvent dans le but de protéger des groupes minoritaires? Il conviendra d'abord de se pencher sur le sort de certaines minorités au sein de la société québécoise : leur protection par le biais de droits individuels est-elle une illustration de l'apothéose de l'individu?

D' autre part, lorsque le droit civil réglemente directement l'organisation et le fonctionnement de groupements, en tant que tels, c'est la loi de la majorité qui l'emporte à première vue, à telle enseigne que l'on peut se poser la question suivante : est-ce la consécration de la dictature de la majorité?

Notre rapport sera donc agencé autour de deux axes : I - Les minorités au sein de la société québécoise : l'apothéose de l'individu? II - Les minorités au sein de groupements de droit civil : la dictature de la majorité? 


\section{Les minorités au sein de la société québécoise : l'apothéose de l'individu?}

Il est clair que les droits garantis par l'État aux minorités - nous écartons volontairement les minorités linguistiques et autochtones - se présentent pour la plupart non pas comme les droits collectifs d'une minorité, mais comme des droits individuels. Il s'agit là sans aucun doute de l'héritage du libéralisme fondé sur le respect des droits et libertés individuels ${ }^{1}$. Tant la Charte canadienne de 1982 [«Chacun a les libertés [...]; chacun a droit»] que la Charte québécoise de 1975 [«Toute personne a droit ...»] épousent cette façon de voir ${ }^{2}$.

La protection d'un groupe minoritaire passe donc par la protection de l'individu, membre de ce groupe. C'était et c'est encore l'individu qui est l'objet de la réglementation du droit civil. On pourrait même argumenter que c'est une conception civiliste qui est consacrée par les textes sanctionnant les droits fondamentaux. Il n'est pas dans notre propos de tenter de définir ce qu'est une minorité ${ }^{3}$. Puisqu'il faut exclure les minorités ethniques et linguistiques ${ }^{4}$, restent surtout les groupes de personnes qui suivent une croyance ou une religion «différente» ou qui épousent un mode de vie significatif «différent». Il est toujours tentant de tomber dans le piège - qu'il convient à tout prix d'éviter - de faire appel au droit public et aux garanties constitutionnelles, même si on ne peut nier que le droit civil baigne dans leurs eaux. Notre propos est donc strictement limité, autant que faire se peut, au droit privé, civil de surcroît. Cependant, il faut souligner que différemment de la Charte canadienne de $1982^{5}$ qui ne vise que les actions gouvernementales et les recours de l'individu à leur encontre, la Charte québécoise ${ }^{6}$ est caractérisée par son ambivalence; elle a non

1. D. Proulx, «Égalité et discrimination dans la Charte des droits et libertés de la personne : étude comparative» (1980) 10 R.D.U.S. 381.

2. J. Deschênes, «Qu'est-ce qu'une minorité?» (1986) 27 C. de D. 254.

3. F. Rigaux, «Mission impossible : la définition de la minorité» (1997) Rev. trim. dr. h. 155; A. Fenet, G. Koubi et I. Schulte-Tenckhoff, Le droit et les minorités, Bruxelles, Bruylant, 2000.

4. Selon les instructions du doyen Mestre.

5. $\quad$ Charte canadienne des droits et libertés de 1982, L.R.C. 1985, App. 11, nº 44.

6. $\quad$ Charte de droits et libertés de la personne, L.R.Q. c. C-12. 
seulement un effet vertical mais son application est horizontale et vise les rapports strictement de droit privé ${ }^{7}$.

Il est cependant inéluctable de devoir traiter des minorités dans le cadre de la discrimination ou du droit à l'égalité, quitte à le faire à travers le prisme des droits individuels des membres de ces minorités; toujours en ayant à l'esprit d'éviter de réitérer ce qui aura été dit lors du colloque de l'Association Henri Capitant qui portait l'an dernier précisément sur la discrimination.

Il faut dire qu'en matière de discrimination, la jurisprudence québécoise (et canadienne) qui s'inspire des arrêts de la Cour suprême a atteint un degré de sophistication et de raffinement extrême. Notre approche ne pourra être que sommaire, voire grossière.

La Charte québécoise contient un chapitre de plus d'une vingtaine de dispositions intitulé «Droit à l'égalité dans la reconnaissance et l'exercice des droits et libertés» dont nous reproduisons ci-dessous les principales :

Art. 10 : [Discrimination interdite] Toute personne a droit à la reconnaissance et à l'exercice, en pleine égalité, des droits et libertés de la personne, sans distinction, exclusion ou préférence fondée sur la race, la couleur, le sexe, la grossesse, l'orientation sexuelle, l'état civil, l'âge sauf dans la mesure prévue par la loi, la religion, les convictions politiques, la langue, l'origine ethnique ou nationale, la condition sociale, le handicap ou l'utilisation d'un moyen pour pallier ce handicap.

[Motif de discrimination] Il y a discrimination lorsqu'une telle distinction, exclusion ou préférence a pour effet de détruire ou de compromettre ce droit.

Art. 12 : [Discrimination dans formation d'acte juridique] Nul ne peut, par discrimination, refuser de conclure un acte juridique ayant pour objet des biens ou des services ordinairement offerts au public.

7. A. Morel, «La coexistence des Chartes canadienne et québécoise : problèmes d'interaction» (1986) 17 R.D.U.S. 49. 
Art. 13 : [Clause interdite] Nul ne peut, dans un acte juridique, stipuler une clause comportant discrimination.

[Nullité] Une telle clause est sans effet.

Art. 15 : [Lieux publics accessibles à tous] Nul ne peut, par discrimination, empêcher autrui d'avoir accès aux moyens de transport ou aux lieux publics, tels les établissements commerciaux, hôtels, restaurants, théâtres, cinémas, parcs, terrains de camping et de caravaning, et d'y obtenir les biens et les services qui y sont disponibles.

Art. 16 : [Non-discrimination dans l'embauche] Nul ne peut exercer de discrimination dans l'embauche, l'apprentissage, la durée de la période de probation, la formation professionnelle, la promotion, la mutation, le déplacement, la mise à pied, la suspension, le renvoi ou les conditions de travail d'une personne ainsi que dans l'établissement de catégories ou de classifications d'emploi.

Art. 17 : [Discrimination par association d'employeurs ou de salariés interdite] Nul ne peut exercer de discrimination dans l'admission, la jouissance d'avantages, la suspension ou l'expulsion d'une personne d'une association d'employeurs ou de salariés ou de tout ordre professionnel ou association de personnes exerçant une même occupation.

La personne qui est victime de discrimination a à sa disposition les recours du droit commun pour faire cesser la discrimination et obtenir réparation du préjudice qui en résulte ${ }^{8}$.

Cependant, la Charte québécoise prévoit en outre un mécanisme particulier qu'il convient de décrire dans ses grandes lignes. Celui qui se prétend victime d'une discrimination peut porter plainte à la Commission des droits de la personne et des droits de la jeunesse $e^{9}$. Le rôle de la Commission est de faire

8. Supra note 6, art. 49. L'article 49, alinéa 2, ajoute qu' «en cas d'atteinte illicite et intentionnelle [à un droit ou à une liberté reconnu par la présente Charte], le tribunal peut en outre condamner son auteur à des dommages punitifs». Il s'agit des dommages exemplaires, sorte de peine privée inspirée du common law.

9. $\quad$ Supra note 6, art. 71 et 74 . 
enquête, d'amener éventuellement les parties à une entente. Elle portera, sans frais pour le contribuable, si elle l'estime adéquat, la cause devant le Tribunal des droits de la personne ${ }^{10}$. Lorsque la personne concernée se prétend victime de discrimination dans le cadre de son emploi, de son logement, ou dans la fourniture de biens et de services, il sera du ressort du Tribunal des droits de la personne de statuer.

Une des particularités du droit de la discrimination est le devoir ou l'obligation d'accommodement dont il convient de dire quelques mots. Cette obligation d'accommodement «raisonnable» s'applique principalement dans les relations d'emploi et de fourniture de biens et de services destinés au public. Elle consiste dans l'obligation pour un contractant de «modifier des normes, des pratiques ou des politiques légitimes et justifiées, qui s'appliquent sans distinction à tous, pour tenir compte des besoins particuliers de certaines minorités, surtout ethniques et religieuses ${ }^{11}$. Ainsi, par exemple, offrir à un employé dont la religion prohibe toute activité un samedi, un autre jour de travail $^{12}$.

Jusqu'ici nous avons décrit des situations où les droits d'une minorité sont exprimés, concrétisés, par l'individu qui en est membre. Il ne s'agit pas toutefois d'une «apothéose» de l'individu, mais d'une prédominance. En effet, le système prévoit l'intervention du groupe minoritaire pour faire valoir ses droits et intérêts, d'abord en raison de voies procédurales particulières, ensuite pour éviter l'application indiscriminatoire de dispositions antidiscriminatoires (sic).

Que ce soit en vertu des règles générales de procédure ou en vertu de la procédure particulière en matière de discrimination devant le Tribunal des droits de la personne, le groupe minoritaire, en tant que tel, peut intervenir. En vertu

10. Ibid., art. 84.

11. J. Woehrling, «L'obligation d'accommodement raisonnable et l'adaptation de la société à la diversité religieuse» (1998) 43 R.D. McGill 325 à la p. 328; C. Brunelle, Discrimination et obligation d'accommodement en milieu de travail syndiqué, Cowansville (Qc), Yvon Blais, 2001.

12. L'affaire Amselem dont nous reparlerons est un bon exemple de la façon habituelle de procéder. 
du Code de procédure civile, c'est autour de la notion d'intérêt ${ }^{13}$ interprétée très libéralement en droit public ${ }^{14}$ que l'opportunité d'une intervention d'un groupe organisé ou représentatif sera jugée ${ }^{15}$.

En vertu de la Charte québécoise, une plainte à la Commission peut aussi «être portée, pour le compte de la victime ou d'un groupe de victimes, par un organisme voué à la défense des droits et libertés de la personne ou au bienêtre d'un groupement ...» ${ }^{16}$ et ces groupes peuvent devenir «parties à la demande» devant le Tribunal des droits de la personne en vertu de l'article 116 al. 2 de la Charte québécoise.

D'autre part, l'article 20 de la Charte québécoise protège un type de discrimination qui n'est pas illicite. Il se lit ainsi ${ }^{17}$ :

Une distinction, exclusion ou préférence [...] justifiées par le caractère charitable, philanthropique, religieux, politique ou éducatif d'une institution sans but lucratif ou qui est vouée exclusivement au bien-être d'un groupe ethnique est réputée non discriminatoire.

La Cour suprême en $1988^{18}$ a jugé qu'il fallait interpréter cette exception de façon libérale : elle limite les droits de certains individus et donne des droits à certains groupes, favorisant ainsi le droit d'association. L'effet de l'article 20 est de protéger certaines minorités en permettant une certaine discrimination. Ce volet de l'article 20 «établit dans des circonstances précises la primauté des droits du groupe sur les droits de l'individu» ${ }^{19}$.

13. Art. 208 C.p.c.

14. Voir, par exemple, Caron c. Canada, [1988] R.J.Q. 2333 (C.A.); Commission scolaire Ancienne-Lorette-Montcalm c. Commission des droits de la personne, [1993] R.D.J. 643 (C.A.).

15. Il faut mentionner les dispositions du Code de procédure civile concernant les actions collectives : art. 999 et $\mathrm{s}$.

16. Supra note 6, art. 74.

17. Dans notre version abrégée.

18. Ville de Brossard c. Commission des droits de la personne, [1988] 2 R.C.S. 279.

19. Ibid. 
C'est précisément ce genre de primauté qui prévaut lorsque le droit civil traite de groupements.

\section{Les minorités au sein de groupements : la dictature de la majorité?}

Lorsque le Code civil traite de groupements de personnes, il fait appel à la règle de la majorité. Cette majorité est parfois qualifiée pour la prise de décisions à conséquences graves.

Ainsi, le conseil de tutelle (ancien conseil de famille) prend ses décisions à la majorité des voix (article 234 C.c.Q.). Il en est de même pour le Conseil d'administration de la personne morale (article 336) ou son assemblée (article 351 C.c.Q.). Il est intéressant de noter que $10 \%$ des voix de l'assemblée suffisent pour requérir la convocation d'une assemblée et que la dissolution de la personne morale exige les $2 / 3$ des voix exprimées (article 356 C.c.Q.).

Le Code civil du Québec a légiféré sur le «contrat d'association» et l'article 2272 est pertinent :

Art. 2272 : Tout membre a le droit de participer aux décisions collectives et le contrat d'association ne peut empêcher l'exercice de ce droit.

Ces décisions, y compris celles qui ont trait à la modification du contrat d'association, se prennent à la majorité des voix des membres, sauf stipulation contraire dudit contrat.

La règle de la majorité se retrouve dans le contexte des décisions relatives à l'administration d'un bien indivis dans le chapitre sur la copropriété par indivision : article 1026. Cependant, les décisions visant à aliéner le bien indivis, à le partager, à le grever d'un droit réel, à en changer la destination ou à y apporter des modifications substantielles sont prises à l'unanimité ( $2^{\mathrm{e}}$ alinéa).

La rigueur de la règle «Nul n'est tenu de demeurer dans l'indivision » a été atténuée dans le nouveau Code civil; par exemple par une convention. Cependant, l'article 1031 prévoit que 
Art. 1031, al. 1 : Malgré toute convention contraire, les trois quarts des indivisaires, représentant 90 p. 100 des parts, peuvent mettre fin à la copropriété indivise d'un immeuble principalement à usage d'habitation pour en établir la copropriété divise.

En matière de copropriété divise d'un immeuble, la règle de la majorité prévaut aussi ${ }^{20}$. Cependant doivent être cités les articles 1097 et 1098 :

Art. 1097 : Sont prises à la majorité des copropriétaires, représentant les trois quarts des voix de tous les copropriétaires, les décisions qui concernent :

$1^{\circ}$ Les actes d'acquisition ou d'aliénation immobilière par le syndicat; $2^{\circ}$ Les travaux de transformation, d'agrandissement ou d'amélioration des parties communes, ainsi que la répartition du coût de ces travaux; $3^{\circ}$ La construction de bâtiments pour créer de nouvelles fractions.

$4^{\circ}$ La modification de l'acte constitutif de copropriété ou de l'état descriptif des fractions.

Art. 1098 : Sont prises à la majorité des trois quarts des copropriétaires, représentant 90 p. 100 des voix de tous les copropriétaires, les décisions :

$1^{\circ}$ Qui changent la destination de l'immeuble;

$2^{\circ}$ Qui autorisent l'aliénation des parties communes dont la conservation est nécessaire au maintien de la destination de l'immeuble;

$3^{\circ}$ Qui modifient la déclaration de copropriété pour permettre la détention d'une fraction par plusieurs personnes ayant un droit de jouissance périodique et successif.

Cet exposé serait incomplet sans quelques mots sur la liberté d'association telle qu'elle se présente dans les relations privées , car bien sûr il s'agit d'une liberté publique constitutionnellement protégée.

Par définition, la liberté d'association ne peut s'exercer seule ! Les recours de celui qui est brimé dans l'exercice de cette liberté sont plutôt des 
recours individuels ${ }^{21}$. Il serait fastidieux de s'aventurer dans les méandres de la législation visant les syndicats. Là aussi, dans le pays fédéral qu'est le Canada, le droit québécois côtoie le droit canadien selon la division des compétences législatives constitutionnelles.

La liberté d'adhérer à un syndicat est même protégée par la sanction d'une infraction criminelle (article 425 Code criminel du Canada). Elle est formellement encadrée par l'article 8 du Code canadien du travail ${ }^{22}$ et par l'article 3 du Code du travail ${ }^{23}$ du Québec...

Même si trois juges minoritaires de la Cour suprême ${ }^{24}$ semblent en douter, la liberté syndicale comprendrait la liberté de ne pas adhérer à un syndicat ${ }^{25}$. Cependant, la contribution financière de tous les salariés de l'unité a été jugée comme conforme à la liberté d'association protégée par l'article $2 \mathrm{~d}$ de la Charte canadienne ${ }^{26} \mathrm{C}$ 'est la «formule Rand» qui est même imposée, au Québec, par voie législative (article 47 C.t.).

En matière de sécurité syndicale, on reconnaît non seulement la clause d'atelier syndical imparfait en vertu de laquelle les travailleurs doivent devenir membres du syndicat après l'expiration d'un certain délai après la date de l'emploi (en général 90 jours), mais la clause d'atelier syndical parfait qui prévoit carrément l'adhésion forcée au syndicat pour travailler, dans certains domaines.

21. Il faut noter aussi le recours qui peut être exercé par le syndiqué contre le syndicat qui aurait commis une faute dans l'exercice de son obligation de représentation syndicale : voir J.-D. Gagnon, «Le devoir de représentation des associations de salariés en droit canadien et québécois» (1981) 41 R. du B. 639.

22. Code canadien du travail, L.R.C. (1985), c. L-2.

23. Code du travail, L.R.Q., c. C-27.

24. Lavigne c. Syndicat des employés de la fonction publique de l'Ontario, [1991] 2 R.C.S. 211. La Cour suprême est d'avis que le droit d'association ne protège pas le droit de négociation collective ni le droit de grève, en vertu de la Charte canadienne.

25. Voir F. Morin et J.-Y. Brière, Le droit de l'emploi au Québec, Montréal, Wilson \& Lafleur, 1998 aux pp. 745 et s. et la décision, pour le moins ambiguë de la Cour suprême du Canada dans R. c. Advance Cutting \& Coring Ltd., [2001] C.S.C. 70.

26. $\quad$ Affaire Lavigne, précitée note 24. 
À titre d'illustration d'une part et de curiosité d'autre part, sont reproduits ici les 2 premiers aliénas de l'article $70 \mathrm{du}$ Code canadien $d u$ travail $^{27}$ :

Art. 70 : (1) [Retenue de la cotisation syndicale] À la demande du syndicat qui est l'agent négociateur des employés d'une unité de négociation, la convention collective conclue avec l'employeur doit contenir une disposition obligeant ce dernier à prélever sur le salaire versé à chaque employé régi par la convention, que celui-ci adhère ou non au syndicat, le montant de la cotisation syndicale normale et à le remettre sans délai au syndicat.

(2) [Objection d'ordre religieux] S'il est convaincu que le refus d'un employé de faire partie d'un syndicat ou de lui verser la cotisation syndicale normale est fondé sur ses croyances ou convictions religieuses, le Conseil peut, par ordonnance, exempter l'employé des dispositions de la convention collective exigeant soit l'adhésion syndicale comme condition d'emploi, soit le versement de la cotisation syndicale normale à un syndicat. L'intéressé est alors tenu de verser, soit directement, soit par prélèvement sur son salaire, un montant équivalent à la cotisation syndicale normale à un organisme de bienfaisance enregistré agréé à la fois par l'employé et le syndicat.

Le second alinéa a été interprété par la Cour d'appel du Manitoba (province qui avec d'autres : - l'Ontario par exemple - a légiféré de la même manière que le fédéral) dans Re Funk and Manitoba Labour Board ${ }^{28}$. Il s'agissait d'un individu appartenant à la religion Mennonite, qui ne prohibait pas directement l'adhésion à un syndicat. Il a été jugé cependant que si la prohibition faisait partie des croyances personnelles du sujet, elle justifiait l'objection d'ordre religieux visée par la loi.

Nous conclurons cet exposé par ce qui aurait pu en servir d'introduction : en faisant état d'une récente décision de la Cour supérieure du

27. Voir aussi Ontario Labour Relations Act, S.0. 1995, C. 1, schedule A, sect. 52.

28. $\quad$ Re Funk and Manitoba Labour Board (1976) 66 D.L.R. (3) 35. 
Québec, décision confirmée en appel le 12 avril 2002, l'affaire Northcrest c. Amselem $^{29}$.

Il s'agit d'un litige entre le Syndicat de copropriétaires d'un immeuble haut de gamme d'une part et, d'autre part, une demi-douzaine d'individus juifs orthodoxes. Le syndicat demande à la Cour supérieure d'ordonner aux intimés de s'abstenir d'ériger et de démolir sur les parties communes à usage exclusif de la copropriété - les balcons, en l'occurrence - des «souccahs». Ces dernières sont des petites cabanes sans toit pour célébrer la fête du «soukot» et sont utilisées pour une période de huit jours à compter du coucher du soleil pour prendre des repas (à défaut d'y dormir vu le climat canadien).

La défense - il faut souligner l'intervention de «The League for Human Rights of B'Nai Brith Canada» - repose essentiellement sur la non-conformité d'une clause contractuelle interdisant toute construction de quelque nature que ce soit sur le balcon avec le droit à la liberté de religion.

Le juge souligne que «pour porter atteinte à la liberté de religion, la clause contractuelle doit, soit directement ou par effet préjudiciable contraindre une personne à agir contrairement à sa conviction religieuse, soit lui interdire un geste considéré obligatoire par sa religion».

En se basant sur une analyse étoffée, le juge accorde l'injonction demandée : la clause attaquée, selon la preuve et les circonstances, n'empêche pas les intimés de remplir leur devoir religieux; de plus, des accommodements raisonnables, à la charge de l'ensemble des copropriétaires, ont été refusés par les requérants.

Nous n'avons pas à nous prononcer sur le fond. Voilà une cause qui touche le droit des contrats (même des biens) et les minorités. On constate : 1 . à l'intérieur d'un contrat multilatéral, l'affaire met en jeu une majorité des copropriétaires opposée à une minorité. Un groupe minoritaire au sein d'un groupement de droit civil; 2. Ce sont les individus, en tant que tels qui sont visés

29. Syndicat Northcrest c. Amselem, [1998] R.J.Q. 1892; P. G. Champagne, «Droits et recours des copropriétaires», dans Développements récents en droit de la copropriété divise, Service de la formation permanente (159), Barreau du Québec, Yvon Blais, 2001, 139. 
et ils font valoir leurs droits individuels (État libéral); 3. Ces individus font partie d'un groupe minoritaire dans la société québécoise (minorité reliée à la religion); 4. Le système procédural en tient compte, car même si les droits sont individuels, on permet à des représentants du groupe visé d'intervenir.

Cette affaire a été confirmée en Cour d'appel ${ }^{30}$ et permission de la Cour suprême du Canada a été par la suite obtenue par les appelants. À suivre, par conséquent. 